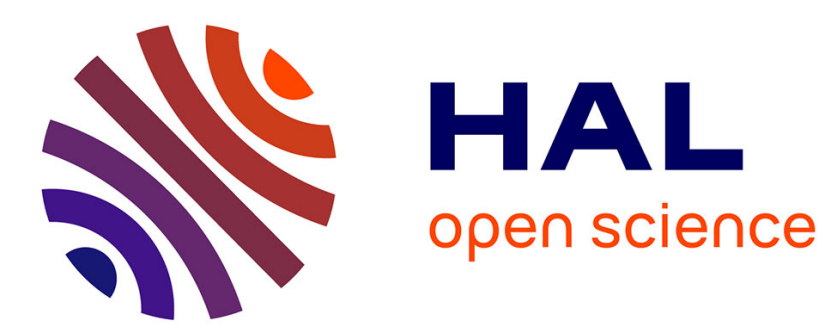

\title{
Sécurité sanitaire des aliments : fausse alerte et vraie crise
}

\author{
Eric E. Cahuzac, Daniel Hassan, Sylvette Monier
}

\section{To cite this version:}

Eric E. Cahuzac, Daniel Hassan, Sylvette Monier. Sécurité sanitaire des aliments: fausse alerte et vraie crise. Economie et Prévision, 2007, 1 (177), pp.55-64. hal-02664693

\section{HAL Id: hal-02664693 https://hal.inrae.fr/hal-02664693}

Submitted on 31 May 2020

HAL is a multi-disciplinary open access archive for the deposit and dissemination of scientific research documents, whether they are published or not. The documents may come from teaching and research institutions in France or abroad, or from public or private research centers.
L'archive ouverte pluridisciplinaire HAL, est destinée au dépôt et à la diffusion de documents scientifiques de niveau recherche, publiés ou non, émanant des établissements d'enseignement et de recherche français ou étrangers, des laboratoires publics ou privés. 


\title{
Sécurité sanitaire des aliments : fausse alerte et vraie crise
}

\author{
Éric Cahuzac $^{(*)}$ \\ Daniel Hassan $^{(*)}$ \\ Sylvette Monier-Dilhan ${ }^{(*)}$
}

Les crises de la dernière décennie (vache folle, poulet aux hormones, dioxine, listéria...) ont hissé la sécurité sanitaire des aliments au niveau des préoccupations majeures des consommateurs des pays développés. La crainte de «mourir empoisonné» succède ainsi à celle ancestrale de «mourir de faim». Elle s'enracine dans un éloignement du consommateur par rapport aux lieux où s'élabore son alimentation. Cet éloignement reflète une industrialisation de la production et de la distribution agro-alimentaire qui, paradoxalement, s'accompagne d'un renforcement croissant des normes sanitaires.

L'objectif de ce travail est de faire l'analyse de l'une de ces crises pour en comprendre certains ressorts. Ces crises sont-elles transitoires ou modifient-elles durablement le comportement du consommateur? Les entreprises mises en cause peuvent-elles s'appuyer sur les consommateurs fidèles au produit ? L'éducation autorise-t-elle plus de distance par rapport aux informations alarmistes? Les messages de réassurance (information positive) parviennent-ils à corriger l'effet d'informations alarmistes? Toutes ces questions, qui tournent autour de la notion de lucidité du consommateur, recoupent les principales interrogations de la littérature récente sur la sécurité sanitaire des aliments. Les réponses apportées peuvent constituer d'utiles repères pour les pouvoirs publics et les entreprises confrontées à de telles crises.

Nous étudions une crise qui a la particularité d'être liée à une fausse alerte. Celle-ci a touché en 1999 une marque connue (notée M) de camembert au lait cru dont les produits ont officiellement été suspectés de contamination par la listéria. En dépit de son absence de fondement et d'un rapide démenti faisant suite à une contre-expertise, cette alerte a été suivie d'effets importants sur la consommation et sur le fonctionnement de l'entreprise (chômage technique). Cette crise fait l'objet d'une double approche. Globale d'une part, pour évaluer son impact sur le marché. Microéconomique d'autre part, l'objectif étant d'identifier les consommateurs sensibles à l'alerte, en fonction des caractéristiques sociodémographiques et des habitudes de consommation. Les données sont celles rassemblées par la Société d'Études de la Consommation, de la Distribution et de la Publicité à partir d'un panel de 8000 ménages dont plus de 6000 achètent du camembert. Sur ce marché, le segment principal est toutefois celui du lait pasteurisé. Le lait cru est un petit créneau (moins de $10 \%$ en valeur) où la marque $M$ représente cependant prés de la moitié des ventes. Nous utilisons les données des années 1998 et 1999.

(*) INRA Économie, Toulouse.

E-mail: Daniel.Hassan@toulouse.inra.fr

E-mail: Sylvette.Monier@toulouse.inra.fr

Les auteurs remercient la Région Midi-Pyrénées pour son aide financière. Ils remercient également les participants aux journées de rencontre du « Pôle Aliment : Sécurité Sanitaire et Santé » ainsi que deux rapporteurs anonymes pour leurs commentaires.

Économie et Prévision

$\mathrm{n}^{\circ} 1772007-1$ 
L'estimation d'une fonction de demande hebdomadaire de camembert sur données agrégées permet de calculer la durée et l'impact de la crise sur la consommation, en faisant la part du trend baissier, des effets saisonniers et de ceux liés aux prix. La durée de la crise ainsi mesurée (14 semaines) est prise comme pas de temps pour l'analyse des comportements des ménages. Celle-ci est réalisée à travers un modèle probit avec biais de sélection expliquant la variable binaire : sortir ou non du marché de la marque incriminée à la période de crise. Les ménages considérés ont réalisé au moins un achat au cours de la période de 14 semaines précédant la crise. Le problème posé par la faiblesse structurelle des fréquences d'achat sur ce segment de marché, qui se traduit couramment chez de nombreux consommateurs par des périodes importantes sans aucun achat, est traité en définissant un benchmark. Nous estimons en effet le même modèle de sortie de marché entre deux périodes indépendantes de la crise.

L'importance de la chute de la demande liée à la crise (-30\% pendant 3 mois) souligne la sensibilité des consommateurs à ce type de menace. Cette réaction de retrait suit de très près la médiatisation de l'alerte. En revanche, les consommateurs ne sont pas immédiatement sensibles aux démentis. On vérifie là un résultat de l'économie de la sécurité sanitaire des aliments concernant le caractère asymétrique des effets de l'information.

Deux éléments permettent cependant de relativiser l'importance de cette crise et d'exhiber une relative lucidité des consommateurs. En effet, cet évènement n'a pas eu de conséquence irréversible : la consommation a retrouvé son niveau initial. Par ailleurs, la crise est restée circonscrite au seul marché de la marque incriminée. Les autres segments du marché des camemberts, en particulier les autres marques de lait cru, n'ont pas été touchés. L'adhésion au lait cru des consommateurs de l'hexagone présente donc une relative stabilité.

L'analyse du comportement individuel des ménages souligne le caractère indifférencié des réactions à la crise. Les personnes âgées, qui sont objectivement parmi les plus fragiles face à la listériose, ne se sont pas reconnues comme sujets à risque. Par ailleurs, contrairement à d'autres crises, l'éducation et la fidélité à la marque n'ont joué aucun rôle modérateur. Ceci peut s'expliquer par l'implication des pouvoirs publics dans le déclenchement de l'alerte. 
La sécurité sanitaire des aliments est un domaine de recherche récent en économie, dont le développement a suivi la montée des inquiétudes des consommateurs au cours des deux dernières décennies. Les travaux réalisés visent à comprendre le fonctionnement des crises, avec un intérêt particulier pour la perception et la gestion du risque par le consommateur. La présente recherche partage ce type de questionnement. Le champ d'observation est une crise survenue en 1999 suite à une alerte à la listéria concernant du camembert au lait cru de marque M. Cette crise repose sur une fausse alerte. Ce type d'incident n'est pas isolé ; il s'est multiplié vers la fin des années quatre-vingt-dix, en relation avec l'inquiétude des pouvoirs publics, dans un contexte marqué notamment par la crise de la vache folle.

La listériose est une maladie grave dont la principale voie de contamination est alimentaire : charcuterie, produits de la mer, produits laitiers... Le risque est augmenté pour certains sujets : femmes enceintes, diabétiques, personnes âgées (multiplié par 7 pour les sujets de plus de 65 ans). La mortalité globale est de 25 à $30 \%$ des personnes atteintes. La réglementation s'est durcie au cours de ces dernières années, suite à une grave épidémie survenue en 1992 (276 malades parmi lesquels 63 morts et 22 avortements). Sévérité accrue des normes et des contrôles se sont traduits en quelques années par une diminution de moitié de l'incidence de cette maladie. L'alerte à la listéria sur la marque $\mathrm{M}$ est déclenchée en mars 1999 par les autorités sanitaires européennes, suite à un contrôle portant sur un lot commercialisé en Belgique. Le communiqué des ministères de la Santé, de l'Agriculture et du Commerce est relayé par la presse ${ }^{(1)}$. Rapidement, des contre-analyses révèlent que le niveau de contamination par la listéria des produits incriminés est largement inférieur au seuil de tolérance établi par la loi française. Malgré ce démenti, on observera bel et bien une crise sur le produit. Cette crise permet d'étudier les réactions des consommateurs à des informations concernant le risque alimentaire.

Dans une recherche sur la première phase de la crise de la vache folle en France, Adda (2001) formule l'hypothèse que les réactions des ménages au risque de contamination obéissent au principe de rationalité du consommateur. Il montre en effet que les plus fortes baisses de consommation ne sont pas le fait des gros mangeurs de viande bovine. Ceux-ci considèrent avoir déjà pris, en raison de leur consommation passée, l'essentiel des risques. De même, les petits acheteurs diminuent peu leurs achats. Ils considèrent que leur faible consommation antérieure les exclut de la population à risque. Par contre, les consommateurs moyens réagissent fortement, pensant pouvoir réduire ainsi le risque. L'auteur s' assure que la réponse des consommateurs n'est pas biaisée par le goût du risque. Pour cela, il utilise comme indicateurs du goût du risque la consommation d'alcool et l'excès de poids : durant la période de crise, ces variables n'influencent pas le comportement d'achat de viande bovine.

L'hypothèse de gestion rationnelle du risque n'est pas admise par tous les auteurs. Viscusi (1990 et 1993) soutient en effet que la perception du risque n'est pas exempte de biais : surestimation de la probabilité d'occurrence de certains risques catastrophiques et peu fréquents (accident d'avion par exemple) et sous-estimation dans le cas contraire. Liu et alii (1998) montrent, à propos d'un incident de contamination du lait par des pesticides, que les effets de l'information sont asymétriques : un message alarmiste a un impact plus fort qu'un message rassurant. Les consommateurs réagissent plus vite dans le premier cas.

Certains travaux soulignent l'importance du caractère répété ou non du message. Ainsi, Brown et Schrader (1990) montrent dans une étude sur le lien entre alimentation et cholestérol, qu'un flux continu d'informations modifie progressivement et durablement les habitudes de consommation. Au contraire, lorsque l'information n'est pas répétée, Dahlgran et Fairchild (2002) montrent que l'effet du message est limité dans le temps. Ce résultat est obtenu en étudiant, à partir d'un modèle de demande, la réponse des consommateurs à une alerte à la salmonellose.

D'autres auteurs analysent le rôle joué par l'éducation sur la perception du risque. Ainsi Herrmann et alii (1997) montrent que les ménages les moins éduqués sont les plus perméables à une information alarmiste. Ils travaillent à partir d'une alerte concernant le caractère cancérigène d'un régulateur de croissance utilisé pour la pomme aux États-Unis au milieu des années quatre-vingt. Cependant, l'éducation n'a pas d'effet unilatéral sur l'attitude face au risque alimentaire. D'autres travaux montrent que, même en présence d'un risque avéré, l'éducation ne s'accompagne pas nécessairement d'une augmentation de la disposition à payer pour réduire ce risque (Henson, 1996 et Roosen et alii, 1998).

La présente étude permet un nouvel examen de certaines des questions abordées dans cette littérature :

- les consommateurs réagissent-ils avec la même rapidité à l'information, indépendamment de son caractère rassurant ou alarmiste ?

- l'effet d'une crise est-il transitoire ou modifie-t-il durablement le comportement des consommateurs?

- l'éducation autorise-t-elle plus de distance par rapport aux informations alarmistes?

- dans le cas d'une crise sans fondement, l'entreprise peut-elle compter sur la fidélité des consommateurs les plus attachés à la marque? 
En première partie, nous étudions l'impact global de la crise en termes d'intensité, de durée et de segments de marché. La deuxième partie est consacrée à l'analyse des réactions individuelles; l'objectif est d'identifier les consommateurs sensibles à l'alerte en fonction des caractéristiques sociodémographiques et/ou des habitudes de consommation. La dernière partie conclut.

\section{Une crise profonde mais limitée dans le temps et sur les produits}

\section{Données}

Ce travail repose sur les données rassemblées par la Société d'Etudes de la Consommation, de la Distribution et de la Publicité (SECODIP) à partir d'un panel de 8000 ménages. Parmi ces ménages, plus de 6000 achètent du camembert (cf. tableau 1). Le marché du camembert se décline en deux segments selon que le lait est pasteurisé ou non. La part de marché du camembert pasteurisé est largement dominante. La marque M représente 44\% des ventes en volume du segment des camemberts au lait cru.

Les panélistes enregistrent, au cours de l'année civile, chacun de leurs achats destinés à la consommation à domicile. Ces enregistrements portent sur les caractéristiques des achats (marque, poids, prix, enseigne, date d'achat...). De plus, SECODIP dispose d'un fichier " ménages » dans lequel sont renseignées certaines caractéristiques sociodémographiques (âge, revenu, lieu de résidence, niveau d'éducation, composition de la famille...). Nous utilisons les données des années 1998 et 1999. Le tableau ci-dessous indique, pour le marché du camembert et pour chaque segment de ce marché, l'effectif des ménages et leur consommation moyenne pour chacune de ces deux années.

Tableau 1 : nombre d'acheteurs et consommation par ménage sur les différents segments du marché du camembert

\begin{tabular}{|c|c|c|}
\hline & 1998 & 1999 \\
\hline \multicolumn{3}{|l|}{ Marché du camembert } \\
\hline Nombre de ménages acheteurs & 6398 & 6004 \\
\hline Consommation par ménage (kg) & $3,326 \mathrm{~kg}$ & $3,204 \mathrm{~kg}$ \\
\hline \multicolumn{3}{|c|}{ Marché des autres camemberts au lait cru } \\
\hline Nombre de ménages acheteurs & 1637 & 1539 \\
\hline Consommation par ménage (kg) & $0,556 \mathrm{~kg}$ & $0,656 \mathrm{~kg}$ \\
\hline \multicolumn{3}{|c|}{ Marché du camembert de la marque M (lait cru) } \\
\hline Nombre de ménages acheteurs & 854 & 727 \\
\hline Consommation par ménage (kg) & $0,851 \mathrm{~kg}$ & $0,728 \mathrm{~kg}$ \\
\hline \multicolumn{3}{|c|}{ Marché des camemberts au lait pasteurisé } \\
\hline Nombre de ménages acheteurs & 5825 & 5463 \\
\hline Consommation par ménage $(\mathrm{kg})$ & $3,070 \mathrm{~kg}$ & $2,988 \mathrm{~kg}$ \\
\hline
\end{tabular}

\section{Identification de la crise}

Le graphique 1 retrace l'évolution des consommations hebdomadaires de camembert de marque $\mathrm{M}$ en 1998 et 1999 . Les données sont exprimées en indices (indice $100=$ moyenne des semaines 2, 3 et 4 en 1998 et en 1999). Ce graphique met en évidence la baisse de consommation correspondant à la crise. Cette crise semble démarrer immédiatement après l'alerte (semaine 13). En revanche, la réaction des consommateurs aux résultats de la contre-analyse attestant de l'absence de listéria n'est pas instantanée. Bien que ces résultats, relayés par la presse ${ }^{(2)}$, aient été disponibles une quinzaine de jours après le début de la crise, celle-ci se prolonge encore une douzaine de semaines.

\section{Graphique 1 : achats hebdomadaires de camembert de marque $M$ en 1998 et 1999}

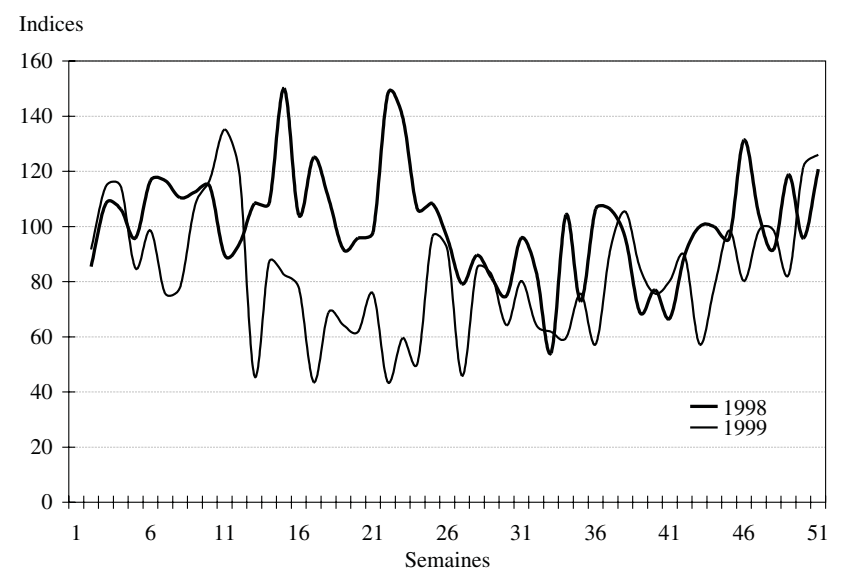

Pour déterminer précisément la durée de la crise nous régressons le logarithme de la demande hebdomadaire ${ }^{(3)}$ globale de camembert (notée $\ln Q_{t}$ ) sur :

- des variables dichotomiques (indicatrice marque $\mathrm{M}$, indicatrice autres camemberts au lait cru) qui permettent de moduler la constante (qui se réfère au camembert au lait pasteurisé) pour prendre en compte les différences de consommation sur chaque segment de marché ;

- année qui permet de pallier un problème d'échantillonnage lié à la réduction de l'effectif du panel d'environ 10\% en 1999 par rapport à 1998 ;

- les variables de saison, notamment été qui capture la diminution de consommation liée à la période estivale ;

- le prix relatif du camembert $\mathrm{M}$ par rapport à tous les autres camemberts ;

- des variables dichotomiques crise sur chacun des trois segments du marché. Ces variables prennent la valeur 1 si le segment de marché subit la crise, 0 sinon.

L'objet de l'estimation est de tester différentes hypothèses concernant les dates de début et de fin de période de crise. Les critères retenus sont la mesure de la qualité globale de la régression (le coefficient de détermination $R^{2}$ ) et celle de précision du 
coefficient de la variable crise ( $t$ de Student). Sur le segment de la marque $M$, ces deux mesures sont simultanément les meilleures pour la période qui débute fin mars 1999 (semaine 13) et s'achève début juillet (semaine 26) : la crise dure un trimestre (14 semaines).

Seul le marché de la marque $\mathrm{M}$ est confronté à la crise. En effet, les coefficients de crise sur autre Lait Cru et crise sur Pasteurisé ne sont pas significativement différents de zéro. Ce résultat reste valable lorsqu'on envisage des durées de crise différentes de celle observée sur le produit incriminé. Les résultats sont indiqués au tableau 2. Le graphique présenté en annexe confirme que la crise n'est pas perceptible lorsque l'on considère l'ensemble du marché du camembert.

La crise se traduit par une baisse de la demande hebdomadaire pour la marque $\mathrm{M}$ de $33 \%$ ( $c f$. coefficients $d u$ tableau 2). Ce résultat n'est pas imputable à une augmentation du prix relatif de la marque $\mathrm{M}$ suite à des offres promotionnelles de la part des autres marques par exemple. On constate au contraire que ce prix relatif a baissé durant la période de crise de $7 \%$, suite à une diminution du prix du camembert $\mathrm{M}$.

Tableau 2 : intensité de la crise

\begin{tabular}{|l|c|}
\hline \multicolumn{1}{|c|}{ Variables } & Coefficients \\
\hline Constante & $6,02 * * *$ \\
Indicatrice de marque M & $-3,29 * * *$ \\
Indicatrice d'autres camemberts au lait cru & $-1,93^{* * *}$ \\
Effet année & $-0,16^{* * *}$ \\
Eté & $-0,21^{* * *}$ \\
Automne & $-0,08^{* * *}$ \\
Hiver & 0,04 \\
Prix relatif & $-0,06$ \\
Crise M & $-0,41^{* * *}$ \\
Crise sur autre Lait Cru & 0,04 \\
Crise sur Pasteurisé & $-0,01$ \\
\hline
\end{tabular}

$* * * \mathrm{p}<0,001 ; * \mathrm{p}<0,05$

\section{Consommateurs : des réactions peu différenciées}

Pour étudier les réactions des consommateurs à l'alerte, nous analysons le comportement des ménages présents sur le marché de la marque $M$ avant la crise. Nous considérons qu'un ménage était présent s'il a acheté au moins un camembert de cette marque au cours des 14 semaines précédant l'alerte, durée qui correspond à celle de la crise. Par rapport à la période qui précède la crise (T-1), la baisse de la demande pour la marque $M$, en période de crise $(T)$, est de $40 \%$. Ce chiffre corrobore l'évaluation obtenue précédemment à partir de la régression. Cette diminution de la demande est liée au choix des consommateurs et non à la décision des offreurs : en effet, la marque incriminée a toujours été présente sur les linéaires. La majeure partie (4/5) de cette baisse est imputable à des abandons de consommation : les ménages concernés n'effectuent aucun achat de camembert $\mathrm{M}$ durant la période de crise. L'autre partie est due à des réductions de consommation. Nous étudions le comportement du premier type de ménages.

Sur le marché du camembert au lait cru, la plupart des consommateurs ont des fréquences d'achat faibles $^{(4)}$. De nombreux consommateurs ne sont pas présents durant deux périodes consécutives ${ }^{(5)}$. Pour appréhender la spécificité de la crise, nous comparons les abandons entre les périodes $\mathrm{T}-1$ et $\mathrm{T}$ à ceux intervenus entre les périodes $\mathrm{T}-2$ et $\mathrm{T}-1$. En procédant ainsi nous nous donnons une situation de référence (cf. tableau 3 ). La crise se traduit par une forte augmentation de ces abandons $(+17 \%)$. Toutefois, cette hausse semble ne pas affecter tous les consommateurs. Seuls les ménages qui abandonnent uniquement la marque $\mathrm{M}$ et restent présents sur l'un des autres segments du marché paraissent être sensibles à l'alerte (cf. ligne $3 \mathrm{du}$ tableau 3). Le pourcentage des ménages qui abandonnent tout le marché est indépendant de la situation (cf. ligne 4 du tableau 3 ). Nous choisissons d'étudier les effets de la crise selon cette hypothèse. Il convient alors de s'assurer qu'en ne tenant pas compte de la sous-population « abandon de tout le marché » on n'introduit pas un biais.

Tableau 3 : proportions d'individus selon les trois alternatives

\begin{tabular}{|l|c|c|}
\hline \multicolumn{1}{|c|}{ Alternatives } & $\begin{array}{c}\text { Situation de } \\
\text { référence T-2/T-1 }\end{array}$ & $\begin{array}{c}\text { Crise } \\
\text { T-1/T }\end{array}$ \\
\hline Fidélité à la marque M & $44 \%$ & $27 \%$ \\
Abandon de la marque M & $56 \%$ & $73 \%$ \\
$\begin{array}{l}\text { Dont abandon de la marque M } \\
\text { uniquement }\end{array}$ & $36 \%$ & $53 \%$ \\
Dont abandon de tout le marché & $20 \%$ & $20 \%$ \\
\hline
\end{tabular}


Pour corriger d'un éventuel biais, nous adoptons une analyse dérivée des travaux d'Heckman (1979) en estimant par maximum de vraisemblance un modèle probit avec biais de sélection (Van de Ven et Van Pragg, 1981). On suppose que pour un individu donné, la propension à quitter l'ensemble du marché est régie par une équation latente, non observable, de la forme $y_{1 i}^{*}=X_{i}^{\prime} \beta+\varepsilon_{1 i}$ où le vecteur $X$ représente des caractéristiques de l'individu $i, \beta$ le vecteur des paramètres à estimer et $\varepsilon$ le terme d'erreur. Seule la variable binaire $y_{1 i}=\left(y_{1 i}^{*}>0\right)$ est observée. Cette variable signale le fait que l'individu $i$ quitte le marché dans son ensemble. Le comportement d'abandon de la seule marque M est modélisé par une équation d'intérêt également de la forme $y_{2 i}^{*}=Z_{i}^{\prime} \gamma+\varepsilon_{2 i}$. Comme précédemment, seule la variable $y_{2 i}=\left(y_{2 i}^{*}>0\right)$ est observée. Cette variable binaire signale ici le fait que l'individu abandonne la marque M. Les termes d'erreurs, qui sont supposés suivre une loi normale centrée réduite, peuvent être corrélés. Les vecteurs $X$ et $Z$ sont présentés ci-dessous et le tableau 4 résume leurs caractéristiques.

Les caractéristiques sociodémographiques retenues sont : le niveau de diplôme (pas de diplôme, BEPC, baccalauréat ou diplôme supérieur), l'âge et le positionnement du ménage en termes de revenu. Ce positionnement permet de savoir si le ménage se situe au-dessus ou en dessous de la moyenne du revenu par unité de consommation ${ }^{(6)}$. Ces variables sociodémographiques, qui correspondent à la personne de référence, sont introduites comme variables explicatives dans l'équation de sélection et dans l'équation d'abandon de la marque $\mathrm{M}$.

Dans l'équation de sélection nous introduisons trois autres variables :

- les habitudes de consommation sont prises en compte, en premier lieu, par la consommation totale de camembert (pasteurisé et au lait cru, quelle que soit la marque) à la période précédente $\left(Q T o t_{-1}\right)^{(7)}$. En second lieu, nous introduisons le nombre de variétés de fromage consommées durant un an. Il s'agit de synthétiser le goût pour la variété. Le signe attendu de cette variable est positif : l'abandon du marché du camembert est renforcé par l'existence de substituts ;

- une variable «taux de présence» est introduite dans l'équation, pour contrôler si l'abandon est effectif ou si le ménage est «inactif» au sens de Sécodip ${ }^{(8)}$.

L'équation de sélection latente retenue est :

$$
\begin{aligned}
y_{1 i}^{*}= & \beta_{0}+\beta_{1} \text { BEPC }+\beta_{2} \text { Bac }+\beta_{3} \text { DiplômeSup } \\
& +\beta_{4} \text { âge }+\beta_{5} \text { Position Revenu }+\beta_{6} \text { Qtot }_{-1} \\
& +\beta_{7} \text { VariétésFrom }+\beta_{8} \text { Présence }+\varepsilon_{1 i}
\end{aligned}
$$

L'individu de référence $\left(\beta_{0}\right)$ a un revenu inférieur au revenu moyen, il n'est pas diplômé, sa consommation totale de camembert et le nombre de personnes au foyer sont les valeurs moyennes sur l'échantillon.

Outre les variables sociodémographiques, l'équation relative à l'abandon de la marque $M$ prend en compte :

- les habitudes de consommation au travers des achats de la période précédente pour chaque type de camembert. Pour la marque $M$, nous supposons que leur réaction à la crise est fonction de leur consommation. Pour cela, nous distinguons les petits consommateurs (un seul camembert consommé sur la période), les consommateurs moyens (2, 3 ou 4 camemberts) et les gros consommateurs ( 5 et plus). Sur les autres segments du marché nous utilisons la quantité consommée : celle de camemberts au lait cru autres que la marque $\mathrm{M}\left(Q A L C_{-1}\right)$ et celle des camemberts au lait pasteurisé $\left(\right.$ QPast $\left._{-1}\right)$;

- de plus, nous introduisons deux variables pour évaluer les effets de substitution dus à la crise : les variations de consommation de camembert pasteurisé $(\triangle Q A L C)$ et celles de camembert au lait cru ( $\triangle Q P$ ast $)$ entre deux périodes consécutives.

L'équation relative à l'abandon de la marque $\mathrm{M}$ s'écrit :

$$
\begin{aligned}
y_{2 i}^{*} & =\gamma_{0}+\gamma_{1} \text { BEPC }+\gamma_{2} \text { Bac }+\gamma_{3} \text { DiplômeSup } \\
& +\gamma_{4} \hat{a g e}+\gamma_{5} \text { Position Revenu }+\gamma_{6} \text { PetitConso } \\
& +\gamma_{7} \text { GrosConso }+\gamma_{8} \text { QALC }{ }_{-1}+\gamma_{9} \Delta Q A L C \\
& +\gamma_{10} \text { QPast }_{-1}+\gamma_{11} \text { DQPast }+\varepsilon_{2 i}
\end{aligned}
$$

L'individu de référence $\left(\gamma_{0}\right)$ a un revenu inférieur au revenu moyen, il n'est pas diplômé, c'est un consommateur moyen de $\mathrm{M}$, son âge et ses consommations correspondent aux valeurs moyennes de l'échantillon.

Les statistiques descriptives sont indiquées au tableau 4 ci-dessous.

Le tableau 5 donne les résultats de l'estimation de l'équation d'abandon de l'ensemble du marché du camembert. Pour cette équation, nous nous intéressons uniquement à la significativité des coefficients $\beta$ et à leur signe. Le principal intérêt de cette étape est de tester l'indépendance des termes d'erreur $\varepsilon_{1}$ et $\varepsilon_{2}$ des deux équations.

Le coefficient de corrélation des termes d'erreur n'est pas significativement différent de 0 , quelle que soit la période : le comportement des ménages qui abandonnent l'ensemble du marché n'est pas motivé par la crise. Ces ménages, qui représentent $20 \%$ de population, que ce soit en période de crise ou non, sont de petits consommateurs qui auraient quitté le 
Tableau 4 : statistiques descriptives

Les quantités sont en kilogrammes

\begin{tabular}{|c|c|c|}
\hline Variables & Période avant la crise & Période de crise \\
\hline Effectif & 349 & 414 \\
\hline \multicolumn{3}{|c|}{ Caractéristiques socio- démographiques } \\
\hline Sans diplôme & $38 \%$ & $41 \%$ \\
\hline $\mathrm{BEPC}$ & $22 \%$ & $21 \%$ \\
\hline $\mathrm{Bac}$ & $21 \%$ & $21 \%$ \\
\hline Diplôme supérieur & $19 \%$ & $17 \%$ \\
\hline Âge (ans) & 50,4 ans & 51,4 ans \\
\hline $\begin{array}{l}\% \text { de la population dont le revenu est supérieur au revenu moyen par unité de } \\
\text { consommation }\end{array}$ & $36,7 \%$ & $35,3 \%$ \\
\hline \multicolumn{3}{|c|}{ Habitudes de consommation } \\
\hline \multicolumn{3}{|c|}{ Marque $\mathrm{M}$} \\
\hline & \multicolumn{2}{|c|}{$\begin{array}{l}\text { Types de consommateur } \\
\text { (fonction des achats à la période précédente) }\end{array}$} \\
\hline Petits & $62 \%$ & $60 \% \%$ \\
\hline Moyens & $30 \%$ & $27 \%$ \\
\hline Gros & $8 \%$ & $8 \%$ \\
\hline \multicolumn{3}{|c|}{ Consommation de camemberts au lait crû, autres que la marque $\mathrm{M}$} \\
\hline Quantité consommée à la période précédente & 0,38 & 0,35 \\
\hline Écart de consommation entre les périodes $\mathrm{T}$ et $\mathrm{T}-1$ & $-0,01$ & $-0,01$ \\
\hline \multicolumn{3}{|c|}{ Consommation de camemberts au lait pasteurisé } \\
\hline Quantité consommée à la période précédente & 0,53 & 0,67 \\
\hline Écart de consommation entre les périodes T et T-1 & 0,02 & $-0,02$ \\
\hline \multicolumn{3}{|c|}{ Consommation totale de camembert } \\
\hline À la période précédente & 1,44 & 1,49 \\
\hline \multicolumn{3}{|c|}{ Autres variables } \\
\hline Nombre de variétés de fromages consommés sur un an & 9,41 & 8,4 \\
\hline Présence (nombre de semaines) & 9,13 & 7,43 \\
\hline
\end{tabular}

Tableau 5 : équation de sélection

\begin{tabular}{|c|c|c|}
\hline \multirow[t]{3}{*}{ Variables } & Non crise & Crise \\
\hline & \multicolumn{2}{|c|}{$\begin{array}{c}\text { Caractéristiques } \\
\text { sociodémographiques }\end{array}$} \\
\hline & Coefficients & Coefficients \\
\hline BEPC & $-0,06$ & $-0,18$ \\
\hline $\mathrm{Bac}$ & 0.21 & 0,05 \\
\hline Diplôme supérieur & $-0,02$ & $-0,31$ \\
\hline Âge & 0,002 & $-0,004$ \\
\hline \multirow[t]{2}{*}{ Niveau de revenu } & 0,7 & $-0,35$ \\
\hline & \multicolumn{2}{|c|}{ Habitudes de consommation } \\
\hline $\begin{array}{l}\text { Quantité totale de camemberts } \\
\text { QTot }_{-1}\end{array}$ & $-0,79 *$ & $-1,10^{*}$ \\
\hline \multirow[t]{2}{*}{$\begin{array}{l}\text { Nombre de variétés de fromages } \\
\text { consommés annuellement }\end{array}$} & $-0,09 *$ & $-0,04$ \\
\hline & \multicolumn{2}{|c|}{ Taux de présence } \\
\hline $\begin{array}{l}\text { Taux annuel de présence dans } \\
\text { le fichier Sécodip }\end{array}$ & $-0,15^{*}$ & $-0,11 *$ \\
\hline Constante & $2,07 *$ & $2,22 *$ \\
\hline Rho & 0,27 & 0,19 \\
\hline Log-vraisemblance & & \\
\hline Effectif & 349 & 414 \\
\hline Observations censurées & 66 & 82 \\
\hline
\end{tabular}

marché même en l'absence de crise. Toutes marques confondues, ces ménages achètent, avant la crise, en moyenne 2 camemberts alors que les autres ménages en consomment 7. La significativité des coefficients liés aux habitudes de consommation et au taux de présence corrobore cette affirmation.

Les résultats du modèle probit concernant l'abandon ou non de la marque $\mathrm{M}$ figurent au tableau 6 . Nous présentons les résultats des estimations (colonnes 1 et 3 ) ainsi que les effets marginaux (colonnes 2 et 4 ). Compte tenu de la non-linéarité du modèle estimé et du risque de variation de ces effets selon le point où on les calcule, nous les calculons comme la moyenne des effets marginaux individuels (Chamberlin) et non pas au point moyen.

Légende : ${ }^{*} \mathrm{p}<0,05$. 
Tableau 6 : modèle probit sur l'abandon de la marque $M$; période de référence et période de crise

\begin{tabular}{|c|c|c|c|c|}
\hline Variables & Coefficients & Effets marginaux (\%) & Coefficients & Effets marginaux \\
\hline \multirow[b]{2}{*}{ Constante } & \multicolumn{2}{|c|}{ Avant crise } & \multicolumn{2}{|c|}{ Crise } \\
\hline & $-0,62$ & & 0,17 & \\
\hline & \multicolumn{4}{|c|}{ Caractéristiques sociodémographiques } \\
\hline BEPC & 0,01 & 0,3 & $-0,00$ & $-0,00$ \\
\hline $\mathrm{Bac}$ & 0,01 & 0,2 & 0,21 & 6,4 \\
\hline Diplôme supérieur & $-0,13$ & $-4,3$ & 0,15 & 5 \\
\hline Âge & 0,01 & 0,2 & $-0,01$ & $-0,2$ \\
\hline \multirow[t]{3}{*}{ Niveau de revenu } & $-0,42 *$ & $-14,4^{*}$ & $-0,12$ & $-3,9$ \\
\hline & \multicolumn{4}{|c|}{ Habitudes de consommation } \\
\hline & \multicolumn{4}{|c|}{$\begin{array}{l}\text { Types de consommateur de la marque } \mathrm{M} \text { (fonction des achats à la période } \\
\text { précédente) Individu de référence : acheteur moyen }\end{array}$} \\
\hline Petits consommateurs & $0,65^{*}$ & $23,1^{*}$ & $0,68^{*}$ & $23,7^{*}$ \\
\hline Gros consommateurs & $-1,04 *$ & $-31,7 *$ & $-0,78^{*}$ & $-27,6^{*}$ \\
\hline & \multicolumn{4}{|c|}{ Quantité consommée période précédente } \\
\hline Camemberts au lait crû, autres que la marque M & $-0,13$ & $-4,4$ & $-0,06$ & $-1,9$ \\
\hline Camembert au lait pasteurisé & $0,19 *$ & $6,5^{*}$ & $0,21 *$ & $6,8^{*}$ \\
\hline & \multicolumn{4}{|c|}{ Effets de substitution } \\
\hline Camemberts au lait crû, autres que la marque M & 0,8 & 2,6 & $0,44^{*}$ & $14,0^{*}$ \\
\hline Camembert au lait pasteurisé & 0,18 & 6,1 & $0,35^{*}$ & $11,0^{*}$ \\
\hline $\begin{array}{l}\text { Probabilité d'abandon de la marque M par l'individu de } \\
\text { référence }\end{array}$ & & 0,37 & & 0,51 \\
\hline
\end{tabular}

Légende : ${ }^{*} \mathrm{p}<0,05$.

La crise se traduit principalement par une augmentation de la probabilité d'abandon de la marque $M$ par 1'individu de référence. Cette probabilité estimée passe de $37 \%$ à $51 \%$.

Le niveau de diplôme et l'âge n'influencent pas les décisions de consommation, que l'on soit ou non en période de crise. Parmi les variables sociodémographiques, seul le revenu a un impact différencié. En période normale, un ménage aisé a une probabilité de sortir du marché du camembert M inférieure à celle d'un ménage dont le revenu est plus faible. Ce résultat n'est pas surprenant car il s'agit d'un camembert relativement coûteux. En période de crise aucun effet revenu n'est observé.

Les habitudes de consommation ne changent pas les comportements d'achat en période de crise, mais des substitutions s'opèrent :

- l'effet des habitudes de consommation du bien M (petit, moyen ou gros consommateur) sur la probabilité d'abandonner est sensiblement le même aux deux périodes. En effet, le test de Chow ne rejette pas l'égalité de ces coefficients. En période de crise, la fidélité à la marque n'a donc pas joué. Le tableau 7 indique, toutes choses égales par ailleurs, les probabilités de quitter le marché de $\mathrm{M}$ selon la fidélité à la marque ${ }^{(9)}$. La probabilité d'abandon augmente de $14 \%$ pour tous les types de consommateurs. Ainsi, les gros consommateurs qui, hors crise étaient quasi-captifs, ne le sont plus : leur probabilité de quitter le marché de M passe de 5,3\% à $19,3 \%$;

- les variables décrivant les habitudes de consommation des autres camemberts au lait cru ou au lait pasteurisé (QALC et QPast) ont des effets qui ne sont pas spécifiques à la crise ;

- par contre, en période de crise, ces produits sont des substituts à la marque $\mathrm{M}$ (cf. les effets marginaux des variables $\triangle Q A L C$ et $\triangle Q P$ ast $)$.

Tableau 7 : probabilité de sortie de M selon la fidélité

\begin{tabular}{|l|c|c|}
\hline & Avant crise & Crise \\
\hline Petits consommateurs de M & $60,1 \%$ & $74,1 \%$ \\
Moyens consommateurs de M & $37,0 \%$ & $51,0 \%$ \\
Gros consommateurs de M & $5,3 \%$ & $19,3 \%$ \\
\hline
\end{tabular}




\section{Conclusion}

L'alerte à la listéria concernant la marque $\mathrm{M}$ fin mars 1999 n'était pas fondée. Pourtant ses effets ont été importants : baisse des ventes de l'ordre de 30 à $40 \%$ (selon les différentes estimations) pendant près de 3 mois. De tels effets peuvent conduire à la faillite d'entreprises plus fragiles économiquement que dans le cas étudié : la marque $\mathrm{M}$ appartient en effet à un groupe qui possède un portefeuille important et diversifié de produits. Cela n'a pas empêché que des salariés de cette entreprise ont été mis en chômage technique.

Pourtant, deux semaines après l'alerte, suite à une contre-expertise, la presse a démenti toute contamination des produits. Malgré cette information rassurante, la crise s'est prolongée durant encore 12 semaines. On observe là un phénomène étudié en économie du risque selon lequel les consommateurs réagissent moins vite à une information positive qu'à une information négative (Liu et alii, 1998).

Cette crise surprend également par son impact peu différencié. Les personnes âgées, qui sont parmi les plus fragiles face à la listériose, ne se sont pas reconnues comme sujets à risque. Par ailleurs, contrairement à d'autres crises, l'éducation et la fidélité à la marque n'ont joué aucun rôle modérateur. Ceci peut s'expliquer par l'implication des pouvoirs publics dans le déclenchement de l'alerte.

La crise n'a cependant pas eu de conséquence irréversible : la consommation a retrouvé son niveau initial. Deux effets ont potentiellement joué. Parallèlement à l'information démentant l'alerte, un mécanisme plus profond a pu fonctionner. En effet, lorsque le message d'alerte est ponctuel, contrairement à une campagne d'information fondée sur la répétition, ses effets se désagrègent progressivement (Dahlgran et Fairchild, 2002). Ce résultat, qui est indépendant du caractère fondé ou non de l'alerte, est utile pour les entreprises et les pouvoirs publics qui doivent gérer des crises concernant la sécurité sanitaire des aliments.

Un autre élément, enfin, relativise l'ampleur de cette crise. Celle-ci est restée circonscrite au seul marché de la marque incriminée. Elle n'a donc pas eu d'effet sur l'adhésion des ménages à la consommation de fromages au lait cru.

\section{Notes}

(1) Le Monde du 26 mars 1999 et Libération du 26 mars 1999.

(2) En particulier le quotidien Libération (9 avril, 10 avril et 16 avril 1999).

(3) Le pas de temps étant la semaine, le nombre d'observations est égal à 100. Pour chaque année, les semaines 1 et 52 ne sont pas retenues car les niveaux de consommation sont systématiquement sous-évalués.

(4) Le délai moyen entre deux achats de camembert M est de 11,5 semaines.

(5) On note à la lecture du tableau 3 qu'en situation normale le pourcentage d'abandons entre deux périodes est élevé (56\%).

(6) L'Insee définit l'unité de consommation de la façon suivante : 0,3 par foyer $+0,7$ par individu de 15 ans ou plus, + 0,5 par individu de moins de 15 ans.

(7) Cette variable n'est pas une variable retardée dans la mesure où elle diffère de l'endogène.

(8) Un ménage est inactif au sens de Sécodip si durant un laps de temps il ne déclare aucun achat.

(9) Ces probabilités sont calculées à partir de celle de l'individu de référence corrigée de la probabilité de quitter le marché en fonction des habitudes de consommation du camembert $\mathrm{M}$. 


\section{Bibliographie}

Adda J. (1999). "Behavior Towards Health Risks: An Empirical Study Using the CJD Crisis as an Experiment" $U C L$ Working Papers 99-02.

Brown D. J. et Schrader L. F. (1990). "Cholesterol Information and Shell Egg Consumption" American Journal of Agricultural Economics. 72(3), pp. 548-555.

Dhlgran R. A. et Fairchild D. G. (2002). " The Demand Impact of Chicken Contamination Publicity - A Case Study" Agribusiness, vol. 18 (4), pp. 459-474.

Jeffrey R. B. et Van Ravenswaay E. O. (1999). "Consumer Demand for Ecolabeled Apples", American Journal of Agricultural Economics, vol. 81, Number 5.

Heckman (1979). "Sample Selection Bias as a Specification of Error", Econometrica vol. 47, n¹, pp. 153-161.

Henson S. (1996). "Consumer Willingness to Pay for Reductions in the Risk of Food Poisoning in the UK "Journal of Agricultural Economics, 47 (3), pp. 403-420.

Herrmann R. O., Warland R. H. et Sterngold A. (1997). "Who Reacts to Food Safety Scares.: Examining the Alar Crisis" Agribusiness 13, pp. 511-520.

Liu S., Huang J-C. et Brown G.L. (1998). "Information and Risk Perception: A Dynamic Adjustment Process" Risk Analysis, vol. 18, pp. 689-700.

Mojduszka E., Caswell J. A., West D. B. et Harris J. M.. In Press. "Changes in the Nutritional Quality of Food Product Offerings and Purchases: A Case Study of Five Categories in the Mid-1990s." USDA, Economic Research Service. Technical Report.

Roosen J., Fox J. A., Hennessy D. A. et Schreiber A. (1998). "Consumers Valuation of Insecticide Use Restrictions: An Application to Apples." Journal of Agricultural and Resource Economics 23, pp. 367-384.

Van de Ven et Van Pragg (1981). "The Demand for Deductibles in Private Health Insurance: A Probit Model with Sample Selection", Journal of Econometrics 17, pp. 229-252.

Viscusi W. K. (1990). "Do Smokers Underestimate Risks?" Journal of Political Economy, vol. 98, n 6 pp. 1253-1269.

Viscusi W.K. (1993). "The Value of Risks to Life and Health," Journal of Economic Literature, vol. XXXI, n ${ }^{\circ} 4$, pp. 1912-1946.
Annexe : évolution de la consommation totale de camembert en 1998 et 1999 ; données hebdomadaires

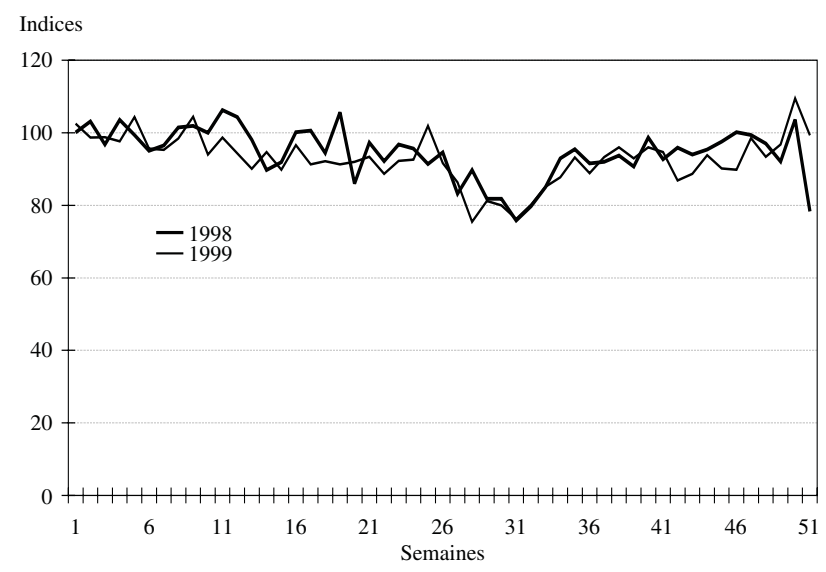

Les données sont exprimées en indices : indice $100=$ moyenne des semaines 2, 3 et 4 en 1998 et en 1999. 